\title{
Distinctive feature enhancement: a review
}

George N. Clements ${ }^{1}$ and Rachid Ridouane ${ }^{1,2}$

${ }^{1}$ Laboratoire de phonologie et phonétique, Sorbonne Nouvelle, France

${ }^{2}$ ENST/TSI/CNRS-LTCI (UMR 5141, Paris)

https://doi.org/10.36505/ExLing-2006/01/0018/000018

\begin{abstract}
We continue the review of some of the basic premises of QuantalEnhancement Theory (Stevens 1972, 1989, etc.) initiated in Clements and Ridouane (2006). While Quantal Theory proposes to account for similarities in feature realisation across speakers and languages, Enhancement Theory proposes to account for regular pat-terns of cross-linguistic variation. In this sense these two theories may be regarded as complementary modules of a more comprehensive feature theory.
\end{abstract}

\section{Expressing variability within quantal theory}

A major challenge to most feature theories comes from the existence of variability in speech output. The realization of a given speech form is never quite the same across utterances, and varies considerably when we take differences in speech rate, style, and speaker into account. It is easily observed that a given distinctive feature may be incompletely realized, or not realized at all, in certain utterances. Indeed, whole segments can be deleted in rapid speech, leaving no traces of their defining features. Furthermore, the realization of a given feature is not necessarily the same from one language to another.

Variability is not in the first instance a problem for Quantal Theory but for the notion of invariance (e.g. Perkell and Klatt 1986). Quantal Theory attempts to provide a basis for explaining why some articulatory and acoustic dimensions are favored over others for feature definitions across languages, but is not logically committed to the claim that all features are realized with their defining properties in all contexts, situations, and languages. The two views are orthogonal to each other: one may maintain a strong form of Quantal Theory while developing independent explanations for variability in feature realization.

Enhancement Theory (e.g. Stevens et al. 1986, Stevens and Keyser 1989, Diehl 1991) has precisely this mission. Starting out from the premise that much crosslinguistic variation is not random but systematic, it attempts to seek the reasons for which some languages systematically prefer one type of feature realization while others prefer another. According to this theory, when the acoustic difference between two sounds is insufficiently great,

ExLing 2006: Proceedings of 1st Tutorial and Research Workshop on Experimental Linguistics, 28-30 August 2006, Athens, Greece 
risking confusion, a supplementary gesture may be introduced to increase the acoustic difference between them. In some cases, this gesture corresponds to a redundant feature, as when the feature [+rounded] is introduced to enhance the difference between back vowels and front vowels (Stevens et al. 1986). This feature has the effect of increasing the auditory difference between front and back vowels by increasing their difference in F2 frequency.

In other cases, the enhancing gesture may be subfeatural, as in the case of the nondistinctive lip-rounding usually added to palato-alveolar sibilants like $/ \check{\mathbf{s}} /$ in English, increasing their auditory difference from alveolar sibilants like $/ \mathrm{s} /$. In this case, too, the enhancing gesture targets the inherent acoustic parameter distinguishing the two sounds and adds a gesture that increases the difference along this parameter. In the case of coronal sibilants, the universal correlate of a post-alveolar [-anterior] fricative appears to be turbulence noise in the region of F3. Adding lip-rounding to such a sibilant accents the spectral prominence in this frequency region and increases its perceptual distance from its [+anterior] counterpart $/ \mathrm{s} /$. Such enhancement would not, of course, be expected in languages that have no contrast of this sort.

Many examples of this sort are provided by Keyser and Stevens (2006). We provide a further example showing that not only feature contrasts, but skeleton-based contrasts between simple and geminate speech sounds can be enhanced in the same way. Tashlhiyt Berber, like many other languages, has a lexical contrast between two types of consonants, singleton and geminate, distinguished phonologically by their association to one vs. two skeletal positions (Dell and Elmedlaoui 1997). As illustrated in (1), this contrast is attested in all positions and concerns all types of consonants:

$$
\begin{array}{llll}
\text { tut } & \text { 'she hit' } & \text { ttut } & \text { 'forget him' } \\
\text { imi } & \text { 'mouth' } & \text { immi } & \text { 'mother' } \\
\text { ifis } & \text { 'jackal' } & \text { ifiss } & \text { 'he is quiet' }
\end{array}
$$

The distinction between simple and geminate consonants is carried not just by duration but by a combination of properties. The primary property is the extra duration of geminates, since this property appears in every context in which the contrast occurs, even in voiceless stops following pause where the closure duration is extra-long even though it has no direct acoustic manifestation (cf. tut vs. ttut above). In addition, the singleton/geminate contrast is enhanced by further acoustic attributes such as shorter preceding vowel duration, higher RMS amplitude, and complete stop closure (Ridouane, in press). These correlates, interpreted as manifestations of greater articulatory energy, serve to enhance the primary feature by contributing additional acoustic properties which increase the perceptual distance between singletons and geminates. These enhancing correlates can take on a distinctive 
function in cases where the primary correlate is not perceptually recoverable. This is, for instance, the case for voiceless stops after pause, where duration differences between singletons and geminates is not detectable by listeners.

\section{Enhancing vs. feature-defining gestures}

Subfeatural enhancing gestures such as these display two properties that distinguish them from basic, feature-defining gestures (Keyser and Stevens 2006). First, unlike feature-defining gestures, subfeatural enhancing gestures are not part of the basic feature definition as such. Evidence that they may have a different status in speech production comes from a consideration of speech error phenomena, in which forms which do not exist in the lexicon can be erroneously generated (e.g. hash or grass becoming the erroneous hass or grash, Fromkin 1973). Such errors can be generated by a transposition of basic feature-defining gestures, in this case the gesture used to distinguish [+anterior] from [-anterior] consonants. However, it appears that no speech error transpositions solely involve enhancing gestures. Thus in a word like sunshine, in which the palato-alveolar feature is enhanced by liprounding, there are no recorded speech errors in which this lip-rounding appears on the initial /s/ while the spread lip configuration expected on the /s/ appears on the palato-alveolar segment.

A related characteristic of subfeatural enhancement gestures is that their implementation appears to be graded. In the example just given, the degree of rounding of the palato-alveolar fricative is weaker and more variable than that of the featurally initiated lip rounding in a [+rounded] vowel such as $/ \mathrm{u} /$. Subfeatural enhancing gestures are non-discrete and continuous, in contrast to feature-defining gestures which are discrete and quantal in nature.

Enhancement displays another rather unexpected property. While feature-defining gestures are often weakened or obliterated in casual speech, enhancement gestures tend to survive intact, preserving underlying distinctions. Here we consider an example involving assibilation. In the historical development of many Bantu languages, the raising of mid vowels to lower high vowels came to threaten the distinction between these vowels and the upper high vowels (we follow the interpretation of Mpiranya 1997). Many of these languages developed strongly assibilated variants of stops before the vowels of the higher series. Following this development, the distinction between upper and lower vowels usually disappeared; but due to assibilation, the distinction between words with earlier upper high vowels and those with earlier lower high vowels persevered. This example is significant in showing that enhancement effects may apply not only to the segments bearing threatened feature distinctions but to segments in their context as well.

In sum, Enhancement Theory offers a basis for understanding some types of regular cross-linguistic variation. Starting from the observation that 
languages tend to preserve useful contrasts, it proposes that supplementary features and gestures may be marshalled to reinforce existing contrasts between two sounds or sound sequences along an acoustic dimension that distinguishes them. Once introduced, these features tend to survive, and may eventually supplant the feature which they originally served to enhance.

\section{Summary}

While Quantal Theory proposes to account for similarities in feature realization across languages, Enhancement Theory proposes to account for (some of) the differences. In this sense they may be regarded as complementary aspects of a more general feature theory.

\section{References}

Clements, G.N. and R. Ridouane. 2006. Quantal Phonetics and Distinctive Features: a Review. Proceedings of ISCA Tutorial and Research Workshop on Experimental Linguistics, 28-30 August 2006, Athens, Greece, pp. xxx-xxx.

Dell. F and M. Elmedlaoui. 1997. Les géminées en berbère. Linguistique Africaine $19,5-55$

Diehl, R.L. 1991. The role of phonetics within the study of language. Phonetica 48, 120-134.

Fromkin, V. A. (ed.) 1973. Speech errors as linguistic evidence. The Hague, Mouton \& Co.

Keyser, S. J. and Stevens, K.N. 2006. Enhancement and overlap in the speech chain. Language 82.1, 33-63.

Mpiranya, F. 1997. Spirantisation et fusion vocalique en bantu: essai d'interprétation fonctionnelle. Linguistique Africaine 18, 51-77.

Perkell, J. and Klatt, D.H. (eds.) 1986. Symposium on Invariance and Variability of Speech Processes. Hillsdale, NJ, Lawrence Erlbaum.

Ridouane, R. in press. Gemination: quality or quantity? Journal of the IPA.

Stevens, K.N. 1972. The quantal nature of speech: Evidence from articulatoryacoustic data. In Denes, P.B. and David Jr., E.E. (eds.), Human Communication, A Unified View, 51-66. New York, McGraw-Hill.

Stevens, K.N. 1989. On the quantal nature of speech. Journal of Phonetics 17, 3-46.

Stevens, K.N. and Keyser, S.J. 1989. Primary features and their enhancement in consonants. Language 65.1, 81-106.

Stevens, K.N., Keyser, S.J. and Kawasaki, H. 1986. Toward a phonetic and phonological investigation of redundant features. In Perkell and Klatt, 426-463. 